\title{
Mutations in Exon 3 of the Glycogen Debranching Enzyme Gene Are Associated with Glycogen Storage Disease Type III That Is Differentially Expressed in Liver and Muscle
}

\author{
Jianjun Shen, ${ }^{*}$ Yong Bao, ${ }^{*}$ Hui-Ming Liu, ${ }^{*}$ Philip Lee, ${ }^{\ddagger}$ James V. Leonard, ${ }^{\ddagger}$ and Yuan-Tsong Chen* \\ $*$ Department of Pediatrics, Division of Medical Genetics, Duke University Medical Center, North Carolina 27710; and ${ }^{\ddagger}$ Great Ormond \\ Street Hospital for Children NHS Trust and Institute of Child Health, University of London, London WC1N 1EH, United Kingdom
}

\begin{abstract}
Glycogen storage disease type III (GSD-III), an autosomal recessive disease, is caused by deficient glycogen debranching enzyme (GDE) activity. Most GSD-III patients are GDE deficient in both liver and muscle (type IIIa), and some GSD-III patients have GDE absent in liver but retained in muscle (type IIIb). The molecular basis for this enzymatic variability is largely unknown. In the present study, the analysis of the GDE gene in three GSD-IIIb patients by single-strand conformation polymorphism (SSCP), DNA sequencing, restriction analysis, and family studies, revealed each of them as being a compound heterozygote for two different mutations. The first mutant alleles in all three patients involved mutations in exon 3 at amino acid codon 6 of the GDE protein. Two had an AG deletion at nucleotides 17 and 18 of the GDE cDNA (17delAG) which resulted in change of subsequent amino acid sequence and a truncated protein (25X); the other had a $\mathrm{C}$ to $\mathrm{T}$ transition at nucleotide 16 of the cDNA which changed a Glutamine codon to a stop codon (Q6X). The 17delAG mutation was also found in 8 of the 10 additional GSD-IIIb patients. The Q6X mutation was found in one of the remaining two GSD-IIIb patients. These two mutations were not found in any of the 31 GSD-IIIa patients, 2 GSD-IIId patients, nor 28 unrelated normal controls. The second mutant alleles in each of the three GSD-IIIb patients were R864X, R1228X, and W680X. The R864X and R1228X were not unique for GSD-IIIb as they were also found in GSD-IIIa patients (frequency of $10.3 \%$ and $5.2 \%$ in Caucasian patients, respectively). Our data demonstrated that both IIIa and IIIb had mutations in the same GDE gene and established for the first time the molecular basis of GSD-III that differentially expressed in liver and muscle. The striking and specific association of exon 3 mutations with GSD-IIIb may provide insight into mechanisms controlling tissue-specific expression of the GDE gene. The identification of exon 3 mutations has clinical significance as well because it distinguished GSD-IIIb from IIIa hence permitting diagnosis from a blood sample rather than a more invasive muscle biopsy. (J. Clin. Invest. 1996. 98:352-357.) Key words: human glycogen debranch-
\end{abstract}

Address correspondence to Y.-T. Chen, M.D., Ph.D., Box 3528, Department of Pediatrics, Duke University Medical Center, Durham, NC 27710. Phone: 919-684-2036; FAX: 919-684-8944.

Received for publication 5 March 1996 and accepted in revised form 7 May 1996.

J. Clin. Invest.

(C) The American Society for Clinical Investigation, Inc.

0021-9738/96/07/0352/06 \$2.00

Volume 98, Number 2, July 1996, 352-357 ing enzyme $\cdot$ glycogen storage disease type III • mutation analysis $\bullet$ tissue-specific gene expression

\section{Introduction}

Glycogen debranching enzyme (GDE), ${ }^{1}$ together with phosphorylase is responsible for degradation of glycogen. The GDE is a $174-\mathrm{kD}$ monomeric protein, with both amylo-1,6glucosidase (EC 3.2.1.33) and 1,4- $\alpha$-D-glucan 4- $\alpha$-D-glycosyltransferase (EC 2.4.1.25) activities at two independent catalytic sites $(1,2)$. Deficiency of GDE activity causes glycogen storage disease type III (GSD-III), an autosomal recessive disease, characterized by hepatomegaly, hypoglycemia, short stature, and in many patients myopathy and cardiomyopathy (3). The variable phenotype is explained by differences in tissue expression of the defective enzyme. Most commonly the enzyme is deficient in both liver and muscle (GSD type IIIa). However sometimes GDE is only deficient in liver and activity in muscle is normal (type IIIb). In rare cases, selective loss of only one of the two DE activities (glucosidase [type IIIc], or transferase [type IIId]) has been demonstrated $(4,5)$.

We and others have isolated the GDE gene and determined its cDNA sequences $(6,7)$. The GDE gene mRNA consists of a 4596-bp coding region and a 2371-bp 3'-nontranslated region. The human gene is localized to chromosome $1 \mathrm{p} 21$ (8). The genomic structure of the human GDE gene has been determined and consists of 35 exons spanning at least $85 \mathrm{~kb}$ of DNA. Analysis of the cDNA sequences and genomic structure indicated that the GDE gene in liver and muscle is encoded by a single gene. The liver and muscle GDE isoform mRNAs are identical except for the sequence present in the 5 '-nontranslated region $(9,10$; Bao et al., manuscript submitted for publication).

The molecular basis of GSD-III and the mechanisms determining the tissue-specific expression of the GDE gene are largely unknown. It has not been understood how one gene, normally expressed in all tissues, can change the expression in different tissues. The presence of patients with defective enzyme that is differentially expressed in liver and muscle make GSD-III an ideal model to study tissue-specific gene expression.

We recently reported the first pathogenic mutation in GSD-IIIa: an insertion mutation (4529insA) in a child with an unusually severe phenotype (11). In this report we focus our study on GSD-IIIb. We have demonstrated a striking and specific association of mutations in exon 3 of the GDE gene with these patients. This finding for the first time establishes the molecular basis of glycogen storage disease type III that is differentially expressed in liver and muscle.

1. Abbreviations used in this paper: GSD-III, glycogen storage disease, type III; GDE, glycogen debranching enzyme; SSCP, singlestrand conformation polymorphism. 


\begin{tabular}{|c|c|c|c|}
\hline Primer & Sequence* & Orientation & cDNA coordinates \\
\hline 1 & $5^{\prime}$ cgatattttaactcctttttg $3^{\prime}$ & Sense & Derived from intron \\
\hline 2 & $5^{\prime}$ agcacgcaaatgagcaaatc3' & Antisense & Derived from intron \\
\hline 3 & $5^{\prime}$ aactttgggcatttttatta $3^{\prime}$ & Sense & Derived from intron \\
\hline 4 & $5^{\prime}$ cctctgaaattgaacctgaag $3^{\prime}$ & Antisense & Derived from intron \\
\hline 5 & $5^{\prime}$ tttgcatatcctgttaaatt $3^{\prime}$ & Sense & Derived from intron \\
\hline 6 & $5^{\prime}$ tctaccaaacaaggcatact $3^{\prime}$ & Antisense & Derived from intron \\
\hline 7 & $5^{\prime}$ 'gggggaaaataagaaaaatg $3^{\prime}$ & Sense & Derived from intron \\
\hline 8 & $5^{\prime}$ caccttgataaatttgtacta3' & Antisense & Derived from intron \\
\hline 9 & 5'CAAAATGGGACACAGTt ${ }^{\ddagger}$ AA3' & Sense & -4 to 15 \\
\hline 10 & 5'AGTTTCTCCATTTCGTTCA3' & Antisense & 35 to 53 \\
\hline 11 & 5'CTGAAGAACGGTTTTACA3' & Sense & 2015 to 2032 \\
\hline 12 & 5'AAATTAACTTCACCTGTG3' & Antisense & 2064 to 2081 \\
\hline 13 & 5'GATCCACATGCACAAGTCG3' & Sense & 2557 to 2575 \\
\hline 14 & 5'GCTAGGCTGCCAGATTTAAA3' & Antisense & 2620 to 2639 \\
\hline 15 & 5'CAGGGCATACAGTTCCGAG3' & Sense & 3637 to 3655 \\
\hline 16 & 5'gttctgtacCTTCGTCCTTC3' & Antisense & $\begin{array}{l}3690 \text { to } 3700+ \\
\text { intron sequence }\end{array}$ \\
\hline
\end{tabular}

*The lowercase letters represent the intron nucleotides.

*The bold lowercase letter $\mathbf{t}$ in primer 9 indicates the mismatched nucleotide.

\section{Methods}

Patients. Our study group included 46 patients with glycogen storage disease type III, for whom both biochemical analysis and clinical data were available; 22 patients were identified through the diagnostic facility for study of glycogen storage disease at Duke University Medical Center, 21 patients were followed at Great Ormond Street Hospital for Children in London, UK, and additional 3 patients' skin fibroblasts were obtained from NIGMS, Human Genetic Mutant Cell Repository, Coriell Institute for Medical Research (cell line numbers: GM00576, GM2523, and GM3390). Glycogen storage disease type III was diagnosed by demonstrating deficient debranching enzyme activity in liver, muscle or mixed leukocytes (or in some patients in more than one tissue) with phosphorylase-limit dextrin used as the substrate (12). In those patients who had not had a muscle biopsy, muscle involvement was inferred from very high activity of plasma creatine kinase (over $3 \times$ upper limit of normal). Blood or skin fibroblasts were the source of genomic DNA isolation.

Mutations were studied in detail in three GSD-IIIb patients (patients 1-3). Data on biochemical analysis and clinical information in these patients have been previously reported (13), debranching enzyme activity was absent in the liver specimen but normal in the muscle specimen. These three patients are now at the age of 25 years (patient 1), 18 years (patient 2), and 41 years (patient 3 ), and continue to show no clinical or laboratory evidence of myopathy or cardiomyopathy.

Single-strand conformation polymorphism analysis. The primers in Table I were used to amplify the coding regions of exon 3 (primers 1 and 2), exon 17 (primers 3 and 4), exon 21 (primers 5 and 6), and exon 28 (primers 7 and 8) of the GDE gene. The DNA fragments were labeled by addition of $0.25 \mu \mathrm{l}$ of $\alpha{ }^{-32} \mathrm{P} \mathrm{dCTP}\left(3,000 \mathrm{Ci} \mathrm{mmol}^{-1}\right.$, $10 \mu \mathrm{Ci} \mu \mathrm{l}^{-1}$ ) into each $25 \mu \mathrm{l}$ of PCR reaction. One microliter of each PCR amplified sample was taken out and added into $10 \mu \mathrm{l}$ of stop solution $(95 \%$ formamide, $10 \mathrm{mM} \mathrm{NaOH}, 0.25 \%$ bromophenol blue, and $0.25 \%$ Xylene cyanol). The sample was denatured for $5 \mathrm{~min}$ in a boiling water bath and placed immediately on ice. $2.5 \mu \mathrm{l}$ of each sample was then loaded onto one or two types of gels: 1) $0.75 \times$ MDE gel (AT Biochemicals, Malvern, PA) containing 5\% (vol/vol) glycerol; 2) $0.75 \times$ MDE gel without glycerol. The gels were electrophoresed at 6-8 W either at room temperature for $18 \mathrm{~h}(\mathrm{~h})$ or at $4^{\circ} \mathrm{C}$ for $36 \mathrm{~h}$. The gels were dried on $3 \mathrm{MM}$ Whatman paper and exposed to Kodak Biomax MR film at $-80^{\circ} \mathrm{C}$.
DNA sequencing. With the SSCP analysis, potential mutations were localized to specific exons within regions of 150-360 bp. To identify the mutations at the nucleotide level, direct sequencing of PCR-amplified genomic DNA fragments without subcloning was performed with the AmpliCycle ${ }^{\mathrm{TM}}$ Sequencing kit (Perkin Elmer, Foster City, CA) using $\gamma^{-32} \mathrm{P}$ dATP $\left(3,000 \mathrm{Ci} \mathrm{mmol}^{-1}, 10 \mu \mathrm{Ci} \mu \mathrm{l}^{-1}\right)$. The same PCR-amplified genomic DNA fragments were also subcloned into $\mathrm{pCR}^{\mathrm{TM}}$ II vector with the TA cloning kit (InVitrogen, San Diego, CA). Plasmid DNA was isolated, alkaline denatured, and mixed with one original PCR primer, then annealed, and sequenced with Sequenase 2.0 (U.S. Biochemicals, Cleveland, OH) using $\left[{ }^{35} \mathrm{~S}\right]$ dATP. Either one or both strands were sequenced with the sequencing primers used in SSCP.

Restriction analysis. Restriction analysis was performed to ensure identified mutations were not PCR artifacts, and to provide a rapid screening for mutation in other patients and family members. Genomic DNA containing each of the suspected mutations was amplified by PCR using a different set of primers. The primers were also listed in Table I. The amplified fragments were digested with their specific restriction enzymes: (a) fragments amplified from exon 3 (using primers 9 and 10) digested with either HinfI for the 17delAG mutation or HincII for the Q6X mutation; $(b)$ fragments amplified from exon 17 (using primers 11 and 12) digested with DraIII; $(c)$ fragments amplified from exon 21 (using primers 13 and 14) digested with $\alpha$-TaqI; and $(d)$ fragments amplified from exon 28 (using primers 15 and 16) digested with DpnII. The digested samples were then electrophoresed on a $12 \%$ polyacrylamide gel. All the restriction enzymes used were purchased from New England Biolabs, Inc. (Beverly, MA).

\section{Results}

\section{Screening mutations with SSCP}

Mutations were studied in detail in three GSD-IIIb patients (patients 1-3). 35 pairs of PCR primers were designed so that they amplified the $5^{\prime}$-nontranslated region as well as coding regions of each of the 35 exons, and all intron-exon junctions, of the GDE gene. The primers used to amplify exons 3, 17, 21, and 28 were listed in Table I. SSCP analysis on MDE gels was followed to detect mobility shift in GSD-III patients. There 

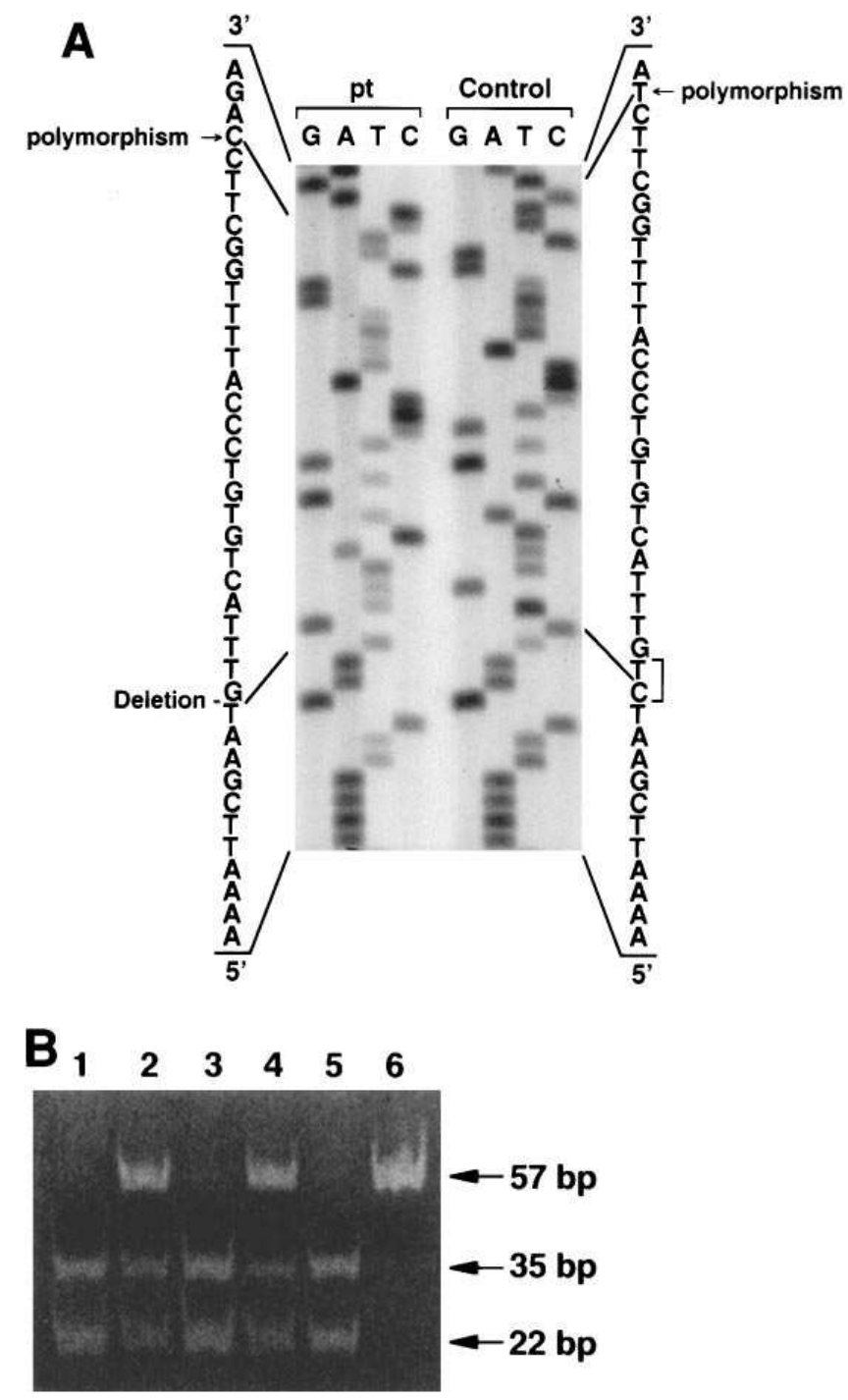

Figure 1. Identification of patient 1's mutation in exon 3. (A) Partial DNA sequences of the GDE gene subclones from normal control and patient $1(\mathrm{pt})$. The sequences shown represented an antisense strand. The GDE gene of patient 1 had an AG deletion (indicated by line) at nucleotides 17 and 18 (17delAG) generating a stop codon at nucleotides 75 to 77 . A normal polymorphism ( $-10 \mathrm{G} / \mathrm{A})$ due to $\mathrm{G}$ to $\mathrm{A}$ change was indicated. $(B)$ HinfI restriction enzyme digestion of PCRamplified genomic DNA fragments in family of patient 1 . Digestion of normal DNA yielded two fragments (35 and $22 \mathrm{bp}$, lane 1) while DNA from patient 1 was only partially digested (lane 2). Lanes 3 and 5 , father and sister of patient 1 . Lane 4 , mother of patient 1 . Lane 6 , a mutant clone from patient 1 as an internal control.

was a two allele polymorphism in exon 3 of these patients and unrelated normal controls. DNA sequencing showed that the polymorphism is due to $\mathrm{G}$ to $\mathrm{A}$ change in $5^{\prime}$-nontranslated region of exon $3(-10 \mathrm{G} / \mathrm{A})$. In addition to the polymorphism, two unique SSCP patterns were also seen in this exon, which represented samples from GSD-IIIb patients (patients 1 and 3). 10 GSD-IIIa patients and one GSD-IIId patient did not have a mobility shift in exon 3 other than those created by $-10 \mathrm{G} / \mathrm{A}$ polymorphism (data not shown).

Further SSCP studies of each of the three GSD-IIIb patients showed additional mobility shifts in $(a)$ exon 21 of pa- tient $1 ;(b)$ exon 28 of patient 2 ; and $(c)$ exon 17 of patient 3. These exons were sequenced.

\section{Identification of mutations}

Patient 1. Sequencing of subclones of patient 1 in exon 3 showed two base pairs adenine and guanine deletion at nucleotides 17 and 18 of the GDE cDNA (17delAG) (Fig. $1 A$ ), which changed the subsequent amino acid sequence and resulted in a stop codon 56 bp down stream in the same exon (25X). The mutation appeared to be heterozygous because only 4 of the 8 subclones sequenced contained the deletion and the other 4 were normal. Direct sequencing showed the AG deletion and a duplicate banding pattern in nucleotides after the deletion confirming the heterozygous nature of the mutation.

The AG deletion (17delAG) abolished an HinfI restriction enzyme site. Using primers 9 and 10 (Table I), a 57-bp fragment of genomic DNA containing the suspected mutation was amplified. After HinfI digestion, the 57-bp fragment was completely digested into two fragments ( 35 and $22 \mathrm{bp}$ ) in a normal control (lane 1 , Fig. $1 \mathrm{~B}$ ) but was only partially digested in patient 1 (lane 2, Fig. $1 B$ ). The pattern of restriction analysis further indicated that patient 1 was heterozygous for the 17delAG mutation and family study indicated that he inherited the deletion from his mother (lane 4, Fig. $1 B$ ). The father and his sister were normal at this position.

An additional SSCP mobility shift in patient 1 was seen in exon 21. Sequencing of subclones of patient 1 in exon 21 showed a $\mathrm{C}$ to $\mathrm{T}$ transition at the position 2590 of the GDE cDNA $(2590 \mathrm{C} \rightarrow \mathrm{T}$ ) (data not shown), which changed an Arginine codon to a stop codon ( $\mathrm{R} 864 \mathrm{X})$. (Our recent data indicate that there are 17 additional amino acids preceding the $\mathrm{NH}_{2}$ terminus of the published GDE sequence [6]. The nucleotide 2590 and amino acid 864 designated here, correspond to the published sequence of nucleotide 2539 and amino acid 847, respectively.) The nonsense mutation appeared to be heterozygous because $(a)$ three of the 6 subclones sequenced were mutant and $(b)$ the mutation produced both $\mathrm{C}$ and $\mathrm{T}$ bands at the same position when PCR products were directly sequenced.

The $\mathrm{R} 864 \mathrm{X}(\mathrm{C} \rightarrow \mathrm{T})$ mutation eliminated an $\alpha$-TaqI restriction enzyme site. Using primers 11 and 12 (Table I), an 83-bp fragment containing the suspected mutation was amplified. After $\alpha$-TaqI digestion, the 83-bp fragment was completely digested into two fragments (50 and $33 \mathrm{bp}$ ) in a normal control but was only partially digested in patient 1 . The pattern of restriction analysis further indicated that patient 1 was heterozygous for the R864X $(\mathrm{C} \rightarrow \mathrm{T})$ mutation and he inherited the point mutation from his father. The sister was not a carrier for either AG deletion or point mutation (data not shown).

Patient 2. Patient 2 had an exon 3 SSCP pattern similar to that of patient 1 . Direct sequencing of the PCR amplified genomic DNA from patient 2 showed the same AG deletion at nucleotides 17 and 18 of the GDE cDNA (17delAG) as patient 1 (data not shown). Patient 2, however, was homozygous for $\mathrm{G}$ at polymorphic site $-10 \mathrm{G} / \mathrm{A}$. Direct sequencing also showed the 17delAG mutation produced duplicate bands in nucleotides after the deletion. This result together with six of the 12 subclones sequenced containing the deletion indicated that the $17 \mathrm{delAG}$ mutation in patient 2 was heterozygous.

Restriction analysis using the same method for patient 1 confirmed that patient 2 was a heterozygote for the AG deletion. The mother of patient 2 was normal at this position, however, the biological father was not available for study. 
An additional SSCP mobility shift in patient 2 was found in exon 28. Sequencing of subclones of patient 2 in exon 28 showed a $\mathrm{C}$ to $\mathrm{T}$ transition at position 3682 of the GDE cDNA $(3682 \mathrm{C} \rightarrow \mathrm{T})$ (data not shown), which changed an Arginine codon to a stop codon (R1228X). The nonsense mutation appeared to be heterozygous because $(a)$ three of the 6 subclones sequenced were mutant and $(b)$ the change produced both $\mathrm{C}$ and $\mathrm{T}$ bands at the same position when PCR products were directly sequenced.

The R1228X $(\mathrm{C} \rightarrow \mathrm{T})$ mutation abolished a DpnII restriction enzyme site. Using primers 13 and 14 (Table I), a 73-bp fragment containing the suspected mutation was amplified. After DpnII digestion, the 73-bp fragment was completely digested into two fragments (42 and $31 \mathrm{bp}$ ) in a normal control but was only partially digested in patient 2 . The pattern of restriction analysis further indicated that patient 2 was heterozygous for the R1228X $(\mathrm{C} \rightarrow \mathrm{T})$ mutation and he inherited this point mutation from his mother (data not shown).

Patient 3. Patient 3 had a unique exon 3 SSCP pattern. Sequencing of subclones of patient 3 revealed a $\mathrm{C}$ to $\mathrm{T}$ transition at the position 16 of the GDE cDNA $(16 \mathrm{C} \rightarrow \mathrm{T}$ ) (data not shown), which changed a Glutamine codon to a stop codon (Q6X). Four of the 8 subclones sequenced were mutant, indicating the change was heterozygous.

The Q6X $(\mathrm{C} \rightarrow \mathrm{T})$ nonsense mutation in patient 3 together with the mismatch in primer 9 (Table I) eliminated an HincII restriction enzyme site. Using primers 9 and 10 (Table I), a 57-bp fragment of genomic DNA containing the suspected mutation was amplified. After HincII digestion, the 57-bp fragment was completely digested into two fragments (40 and 17 bp) in a normal control but was only partially digested in patient 3. The pattern of restriction analysis further indicated that patient 3 was heterozygous for the Q6X $(\mathrm{C} \rightarrow \mathrm{T})$ mutation, and family study indicated that he inherited the point mutation from his father. The mother was normal at this position (data not shown).

An additional SSCP mobility shift in patient 3 was found in exon 17. Sequencing of subclones of patient 3 showed a $G$ to $A$ transition at the position 2039 of the GDE cDNA $(2039 \mathrm{G} \rightarrow \mathrm{A})$, which changed a Tryptophan codon to a stop codon (W680X) (data not shown). The nonsense mutation appeared to be heterozygous because $(a)$ three of the six subclones sequenced were mutant and $(b)$ the mutation produced both $\mathrm{G}$ and $\mathrm{A}$ bands at the same position when PCR products were directly sequenced.

The W680X $(\mathrm{G} \rightarrow \mathrm{A})$ nonsense mutation abolished a DraIII restriction enzyme site. The primers 15 and 16 (Table I) were used to amplify a 67-bp fragment of genomic DNA containing the suspected mutation. After DraIII digestion, the 67-bp fragment was completely digested into 45 and 22 bp fragments in a normal control but was only partially digested in patient 3 . The pattern of restriction analysis further indicated that patient 3 was heterozygous for the W680X $(\mathrm{G} \rightarrow \mathrm{A})$ mutation. The mother of patient 3 was heterozygous for the mutation and the father was normal at this position (data not shown).

\section{Exon 3 mutations in other GSD-III patients}

To determine whether the 17delAG and Q6X mutations occur in other GSD-III patients, we used restriction analysis to screen 43 additional GSD-III patients (10 GSD-IIIb, 31 GSDIIIa, and 2 GSD-IIId) for the presence of these two mutations. Eight of the 10 additional GSD-IIIb patients had the 17 delAG mutation present in the heterozygous form. None of the 31 GSD-IIIa patients, 2 GSD-IIId patients, and 28 normal controls had the deletion. Q6X mutation was found in one of the remaining 2 GSD-IIIb patients in the heterozygous form, but not present in any of the GSD-IIIa patients, GSD-IIId patients, or the 28 normal controls.

\section{R864X, R1228X, and W680X mutations in other GSD-III patients}

To determine whether three point mutations (R864X in patient $1, \mathrm{R} 1228 \mathrm{X}$ in patient 2 , and $\mathrm{W} 680 \mathrm{X}$ in patient 3 ) also occur in other GSD-III patients, we used restriction analysis to screen 43 other GSD-III patients. The R864X point mutation was found in a heterozygous form in 3 GSD-IIIa and 2 other GSD-IIIb patients from 29 Caucasian GSD-III patients, thus the R864X allele accounted for $10.3 \%$ of the Caucasian GSDIII mutant alleles.

Using DpnII restriction enzyme to screen 43 other GSD-III patients, the R1228X mutant allele was found in a heterozygous form in 2 GSD-IIIa patients from 29 Caucasian GSD-III patients. The frequency of this mutation among the Caucasian GSD-III patient population was about $5.2 \%$.

W680X appeared to be a very rare mutation as it was not found in any of the 29 GSD-IIIa, 6 other GSD-IIIb, nor 2 GSD-IIId patients screened.

None of the 20 unrelated controls had any of these mutations.

\section{Discussion}

Although the majority of patients $(\sim 85 \%)$ with GSD-III have GDE deficiency in both liver and muscle, with associated clinical findings of hepatic dysfunction and myopathy, there are $\sim 15 \%$ of patients who have GDE deficiency only in liver with the activity retained in muscle. The molecular basis for this variability is unlikely to be due to defects in tissue-specific isoenzymes encoded by different genes, since there is no clear evidence for the existence of isoforms of the $\operatorname{GDE}(5,14)$. It is also unlikely to be due to defects in a transcription factor since a single transcription factor usually affects the expression of many genes (15). We hypothesized that the two forms of GSDIII are caused by mutations in the same GDE structural gene. This report provides conclusive evidence that GSD-IIIa and IIIb represent allelic heterogeneity of the GDE gene.

Each of the three GSD-IIIb patients studied in detail was a compound heterozygote for two different mutations. The two mutations located on separate chromosomes in each patient were demonstrated by the family studies. Three point mutations, R864X in patient $1, \mathrm{R} 1228 \mathrm{X}$ in patient 2 , and $\mathrm{W} 680 \mathrm{X}$ in patient 3 , all create premature termination codons either in the middle or in the $\mathrm{COOH}$ terminus of the GDE. The mutations resulting in truncated proteins are likely to have deleterious effect on the activity, especially because the $\mathrm{COOH}$ terminus of the GDE contains a putative glycogen binding domain and is believed to be critical for the GDE activity (6). As the R864X and R1228X mutations were also found in GSD-IIIa patients in whom GDE is deficient in both liver and muscle, the mutations must have abolished the GDE activity in both tissues, indicating that the other allele accounts for the differential expression of the GDE. W680X appeared to be a very rare mutation as it was only found in a single patient to date.

The second mutations (17delAG and Q6X) in each of the 3 


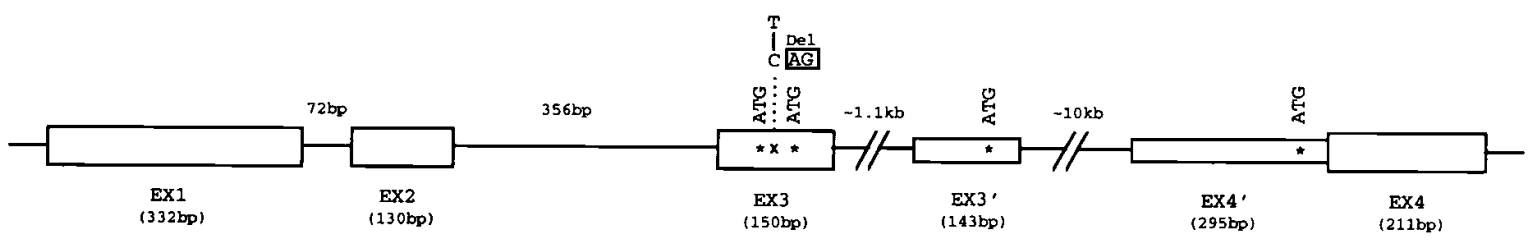

IIVER

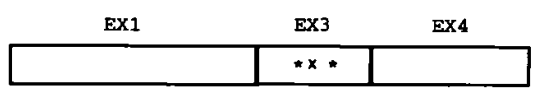

MOSCI:

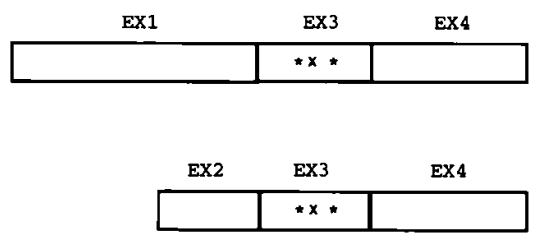

Figure 2. Diagram of $5^{\prime}$-end GDE genomic structure and major isoform mRNAs in liver and muscle. Potential ATG start codons are indicated as * in the diagram. Both exon 3 mutations Q6X and 17delAG are illustrated. The major isoform of GDE mRNA in liver consists of exons 1 and 3. The muscle GDE mRNAs consists of, in addition to the liver isoform, a unique muscle-specific isoform which starts with exon 2.

GSD-IIIb patients resided in exon 3 at amino acid codon 6 (nucleotides 16 to 18 of the GDE mRNA). Both mutations would result in severely truncated proteins having only a few amino acids, which would be unlikely to have GDE activity. The exon 3 mutations were present in 12 of the 13 GSD-IIIb patients (10 with $17 \mathrm{delAG}$ and 2 with Q6X), but not in any of the 31 GSD-IIIa patients, 2 GSD-IIId patients, nor 28 unrelated normal controls. This striking and specific association of exon 3 mutations with GSD-IIIb suggested that exon 3 and its neighboring gene sequences are important in conferring the tissue-specific expression of the GDE.

Only one of the 13 GSD-IIIb patients studied did not have mutations in exon 3 . This patient could have a mutation in the liver-specific promoter and/or enhancer region. Analysis of mutations in GSD-IIIb patients should provide not only a molecular basis of the disease, but also insight into mechanisms controlling tissue-specific expression of the GDE gene.

How can the exon 3 mutations, which create premature stop codons resulting in severely truncated proteins without GDE activity, retain GDE activity in muscle? Liver and muscle GDE isoform mRNAs are identical except for the 5'-nontranslated region. Genomic structure analysis shows that these isoform mRNAs are generated from a single gene by a combined process of differential RNA transcription and processing (Fig. 2). The major isoform of GDE $\mathrm{mRNA}$ in liver is produced by the use of exons 1 and 3 . The muscle GDE mRNAs consists of, in addition to the liver isoform, a unique musclespecific isoform which begins with exon 2 . Exon 3 , which is common for both liver and muscle, is $150 \mathrm{bp}$ in length with 68 bp of $5^{\prime}$-nontranslated sequence, and 82 bp of coding sequence. Translation of GDE protein starts in this exon. Minor isoform mRNAs with the alternative use of exon $3^{\prime}$ or exon $4^{\prime}$ were also observed in both liver and muscle (Fig. 2, and Bao et al., manuscript submitted for publication). Interestingly, these exons also contain ATG start codons with an excellent Kozak sequence (16) in reading frame with the remaining GDE cod- ing sequence. It is possible that because of the nonsense mutations $(a)$ translation of GDE protein begins with a downstream ATG, $(b)$ activation of the minor isoforms with translation starts in exon $3^{\prime}$ or exon $4^{\prime}$, or (c) skipping of exon 3 , with translation starting at a downstream ATG codon. All of these processes would result in a GDE protein which is $\mathrm{NH}_{2}$-terminally truncated. It is conceivable that a lack of the first few $\mathrm{NH}_{2}$-terminal amino acids in a protein as large as GDE (1532 amino acids) has little effect on the GDE activity. At present it is not known whether activation of minor GDE isoform mRNA and/or translation with downstream ATG codon occurs in GSD-IIIb patients at all, and if it occurs, why only in muscle but not in liver. Analysis of GDE mRNA isoforms in muscle from patients with GSD-IIIb and expression studies will provide critical information as to which isoforms are expressed and can also translate into a functional GDE protein; such a muscle sample, however, is not yet available for our analysis.

Previous reports in other systems have documented the presence of regulated expression of exons containing constitutive stop codons. Such exons undergo sex-specific splicing in the sex-lethal gene of Drosophila (17), developmental stagespecific splicing in the rat glutamic acid decarboxylase gene (18) and tissue-specific splicing in the human gene encoding neural cell adhesion molecule (19). Nonsense mutations can also act in cis to alter the usage of alternatively spliced sequences in viral RNA in murine cell (20). It is possible that the severely truncated proteins (5- or 24-amino acid polypeptides) resulting from exon 3 mutations in the GDE gene play a role in conferring the differential GDE expression in liver and muscle. This remains to be tested.

Clinically, GSD-IIIa and GSD-IIIb have different prognoses and outcomes. Myopathy and cardiomyopathy will not occur in GSD-IIIb patients, whereas, they are common in GSD-IIIa patients, which could lead to early death or debilitating adult life. Although muscle involvement could be in- 
ferred from very high levels of plasma creatine kinase, the definitive subtyping of GSD-III requires both liver and muscle biopsy. The striking and specific association of exon 3 mutations with GSD-IIIb could enable a molecular test for subtype of GSD-III.

\section{Acknowledgments}

We thank Thomas Dawson for helpful comments on the manuscript. This work was supported by grants from National Institute of Health DK39078 (to Y.T. Chen) and M01-RR30 (National Center for Research Sources, General Clinical Research Centers program), Muscular Dystrophy Association (to Y.T. Chen) and Pharmacia (to P. Lee).

\section{References}

1. Gordon, R.B., D.H. Brown, and B.I. Brown. 1972. Preparation and properties of the glycogen-debranching enzyme from rabbit liver. Biochim. Biophys. Acta. 289:97-107.

2. Bates, E.J., G.M. Heaton, C. Taylor, J.C. Kernohan, and P. Cohen. 1975. Debranching enzyme from rabbit skeletal muscle; evidence for the location of two active centers on a single polypeptide chain. FEBS Lett. 58:181-185.

3. Chen, Y.-T., and A. Burchell. 1995. Glycogen storage disease. In The Metabolic and Molecular Bases of Inherited Disease. C.R., Scriver, A.L. Beaudet, W.S. Sly, and D. Valle, editors. McGraw-Hill, New York. 935-965.

4. Van Hoof, F., and H.G. Hers. 1967. The subgroups of type III glycogenosis. Eur. J. Biochem. 2:265-270.

5. Ding, J.-H., T. de Barsy, B.I. Brown, R.A. Coleman, and Y.T. Chen. 1990. Immunoblot analyses of glycogen debranching enzyme in different subtypes of glycogen storage disease type III. J. Pediatr. 116:95-100.

6. Yang, B.-Z., J.-H. Ding, J.J. Enghild, Y. Bao, and Y.-T. Chen. 1992. Molecular cloning and nucleotide sequence of cDNA encoding human muscle glycogen debranching enzyme. J. Biol. Chem. 267:9294-9299.

7. Liu, W., M.L. de Castro, J. Takrama, P.T. Bilous, T. Vinayagamoorthy, N.B. Madsen, and R.C. Bleackley. 1993. Molecular cloning, sequencing and analysis of the cDNA for rabbit muscle glycogen debranching enzyme. Arch.
Biochem. Biophys. 306:1-8.

8. Yang-Feng, T.L., K. Zheng, J. Yu, B.-Z. Yang, Y.-T. Chen, and F.-T. Kao. 1992. Assignment of the human glycogen debrancher gene to chromosome 1p21. Genomics. 13:931-934.

9. Bao, Y., B.-Z. Yang, and Y.-T. Chen. 1993. Structural organization of the multifunctional human glycogen debrancher gene. Am. J. Hum. Genet. 53: $662 \mathrm{~A}$

10. Yang, B.-Z., J.-H. Ding, Y. Bao, J.F.M. Eason, and Y.-T. Chen. 1992. Molecular basis of the enzymatic variability in type III glycogen storage disease (GSD-III). Am. J. Hum. Genet. 51:A28.

11. Shen, J., Y. Bao, and Y.-T. Chen. 1996. A nonsense mutation due to a single base insertion in the $3^{\prime}$-coding region of glycogen debranching enzyme gene associated with a severe phenotype in a patient with GSD type IIIa. Hum. Mutat. In press.

12. Brown, D.H., and B.I. Brown. 1966. Enzymes of glycogen debranching: amylo-1,6-glucosidase (I) and oligo-1,4-glucantransferase (II). Methods Enzymol. 8:515-524.

13. Coleman, R.A., H.S. Winter, B. Wolf, J.M. Gilchrist, and Y.-T. Chen. 1992. Glycogen storage disease Type III (glycogen debranching enzyme deficiency): Correlation of biochemical defects with myopathy and cardiomyopathy. Ann. Int. Med. 116:896-900.

14. Taylor, C., A.J. Cox, J.C. Kernohan, and P. Cohen. 1975. Debranching enzyme from rabbit skeletal muscle. Eur. J. Biochem. 51:105-115.

15. Latchman, D.S. 1996. Mechanisms of disease: Transcription-factor mutations and disease. N. Engl. J. Med. 334:28-33.

16. Kozak, M. 1986. Point mutations define a sequence flanking the AUG initiator codon that modulates translation by eukaryotic ribosomes. Cell. 44: 283-292.

17. Bell, L.R., E.M. Maine, P. Schedl, and T.W. Cline. 1988. Sex-lethal, a Drosophila sex determination switch gene, exhibits sex-specific RNA splicing and sequence similarity to RNA binding proteins. Cell. 55:1037-1046.

18. Bond, R.W., R.J. Wyborski, and D.I. Gottlieb. 1990. Developmentally regulated expression of an exon containing a stop codon in the gene for glutamic acid decarboxylase. Proc. Natl. Acad. Sci. USA. 87:8771-8775.

19. Gower, H.J., C.H. Barton, V.L. Elsom, J. Thompson, S.E. Moore, G. Dickson, and F.S. Walsh. 1988. Alternative splicing generates a secreted form of N-CAM in muscle and brain. Cell. 55:955-964.

20. Naeger, L.K., R.V. Schoborg, Q. Zhao, G.E. Tullis, and D.J. Pintel. 1992. Nonsense mutations inhibit splicing of MVM RNA in cis when they interrupt the reading frame of either exon of the final spliced product. Genes \& Dev. 6:1107-1119. 